\title{
Calcul du débit critique de déséquilibre dans les turbomachines à débit partiel. Les déséquilibres thermodynamiques
}

\author{
Calculation of the critical disequilibrium flow \\ in turbine machinery under partial flow conditions. \\ Thermodynamic disequilibria.
}

\author{
Travaux de la Société Hydrotechnique de France
}

Les deux articles qui suivent ne sont pas exactement du modèle de ceux que La Houille Blanche publie habituellement. Ils ne proposent pas de solution à un problème, mais en précisent les données et indiquent une série de directions de recherches.

Deux des groupes de travail constitués au sein de la SHF - l'un sur le fonctionnement des turbomachines en dehors de leur débit nominal, l'autre sur les phénomènes de déséquilibre thermodynamique -- sont arrivés, au terme de plusieurs mois d'échanges et de réflexions, à cerner les problèmes et à préciser les directions dans lesquelles il serait opportun d'engager des recherches. Arrivés à ce point, n'ayant ni la vocation ni les moyens d'entreprendre eux-mêmes ces recherches, ils lancent un appel à la communauté scientifique pour alerter les chercheurs qui auraient, eux, à leur disposition les moyens d'cngager tcllc ou tcllc phase des programmes proposés.

A l'occasion de la réunion-bilan d'une action thématique programmée du CNRS, un premier appel a été lancé le 24 septembre 1980. Ce sont les résumés des deux interventions correspondantes qui sont donnés ci-après.

La Houille Blanche souhaite que ces textes suscitent des échos positifs car, alors, la revue aurait pleinement joué son rôle de carrefour d'échanges et d'informations de la communauté scientifique.

\section{Déséquilibres thermodynamiques}

Un groupe de travail relatif aux phénomènes de déséquilibres thermodynamiques a été constitué dans le cadre de la SHF sous la présidence de M. Lackme, du CENG.

Les phénomènes de déséquilibre se rencontrent dans tous les processus de changement de phase tout spécialement lorsqu'ils sont rapides. Le groupe s'est essen- tiellement intéressé à la vaporisation. Le déséquilibre, dans ce cas, se traduit par une différence entre la température sous laquelle le liquide se met effectivement à bouillir et la température de saturation à la pression correspondante. En d'autres termes, le corps reste liquide dans des conditions où il devrait s'être déjà en partie vaporisé.

Les conséquences de ce phénomène sont particulièrement importantes d'un point de vue pratique dans les applications industrielles suivantes:

- les phénomènes de trempe ou de refroidissement de tôles par pulvérisation qui concernent les industries métallurgiques ;

- la détente adiabatique de liquides, problème particulièrement rencontré dans les réacteurs à eau pressurisée , - la cavitation dans les circuits hydrauliques;

- les évaporateurs à haute ou basse température ; - les circuits cryogéniques.

Le groupe de travail SHF a permis à des spécialistes de chacun de ces domaines de se rencontrer, d'exposer l'état de leurs connaissances et les problèmes non encore résolus.

Malgré la grande diversité des sujets abordés, il est apparu une remarquable convergence dans les préoccupations des participants, la caractérisation des états de déséquilibre :

- problèmes de nucléation homogène et hétérogène ;

- temps caractéristique des phénomènes ;

- valeurs maximales possibles des déséquilibres ;

- prévision des déséquilibres réels ;

- effets de l'hydrodynamique des écoulements, en particulier leur niveau de turbulence sur le phénomène.

Il existe actuellement des réponses partielles à certains de ces points correspondant à des gammes bien définies d'état du fluide ou des conditions expérimentales qui permettent de traiter de manière satisfaisante un certain nombre de problèmes pratiques. 
Cependant, une meilleure connaissance de l'ensemble de ces phénomènes est très souhaitable car ils donnent, sous leur aspect local, une réponse aux conditions initiales de phénomènes macroscopiques que l'on veut, suivant les cas, privilégier ou éviter.

A titre d'exemple, il est très ouhaitable d'avoir, dans le cas du refroidissement par pulvérisation, de l'eau restant sous forme liquide le plus longtemps possible au contact de la paroi chaude. Inversement, dans un évaporateur, les rendements les meilleurs sont obtenus lorsque la vaporisation se produit très près des conditions d'équilibre de saturation.

Le groupe SHF n'ayant ni la vocation, ni les moyens d'entreprendre des études générales d'une telle ampleur, a présenté les grandes lignes de ses activités au cours d'une réunion de l'ATP Thermodynamique du CNRS, en souhaitant que le relais soit pris par des équipes de recherche pour un développement sur le plan fondamental.

$\mathrm{Au}$ cas où de telles actions seraient entreprises, les participants au groupe de travail sont disposés à titre individuel ou collectif à faire part de leur expérience pour la définition et le déroulement des programmes de recherche.

\section{Turbomachines à débit partiel}

Le thème de l'exposé est le suivant : les turbomachines à débit partiel, notamment dans le cas des pompes, ventilateurs et compresseurs centrifuges : Calcul du débit critique de déséquilibre et comparaison avec les résultats expérimentaux.

\section{Introduction et objet des recherches proposées}

La division "Applications industrielles de la mécanique des fluides" de la SHF a créé depuis plus de trois ans, à l'instar d'autres pays, un groupe de travail (GT1) pour l'étude du comportement des turbomachines en fluide incompressible dans les zones de fonctionnement à đébit partiel.

Ce groupe comprend des hydrauliciens et des aérodynamiciens (constructeurs, utilisateurs, universitaires, centres de recherche et d'essais, etc. de langue française).

Ce thème de recherche s'avère du plus grand intérêt tant pour les constructeurs de turbomachines que pour les utilisateurs qui se trouvent de plus en plus confrontés à des instabilités de fonctionnement à débit partiel souvent gênantes et parfois même incompatibles avec la bonne tenue du matériel, dès que le débit de la machine devient inférieur au "débit critique" de déséquilibre. (Voir Figure 1 pour illustration).

Laissant à d'autres organismes mieux placés le soin de progresser pour les compresseurs centrifuges, le groupe de travail s'est attaqué en premier lieu au cas des pompes et des pompes-turbines centrifuges. Ces machines jouent en effet, dans des conditions de plus en plus poussées, un rôle très important dans l'industrie et notamment dans les centrales de production énergétique, tant classiques que nucléaires, ainsi que dans les centrales d'accumulation d'énergie. La meilleure con-

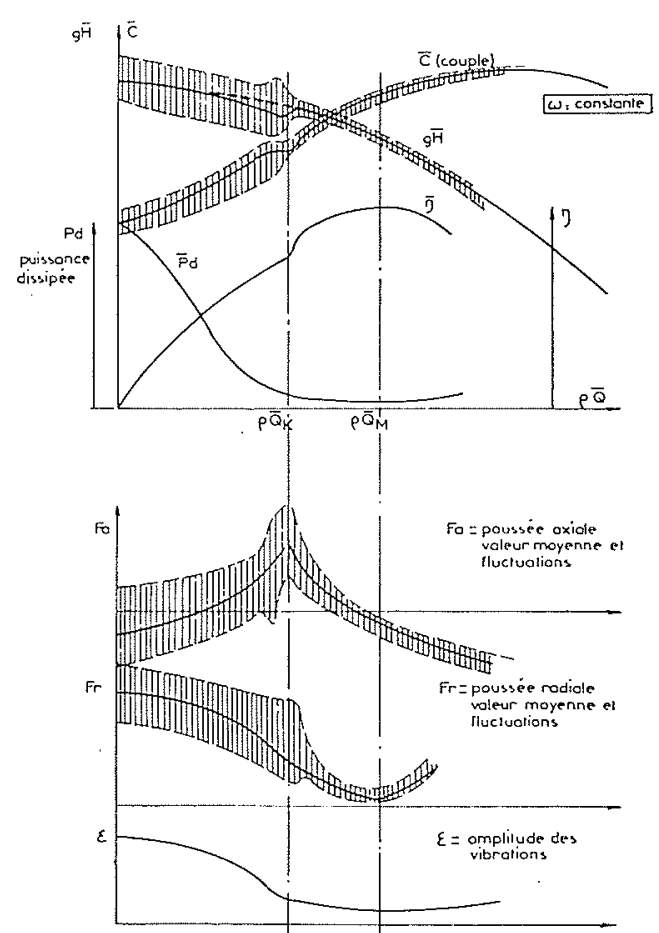

Figure 1 - Figure schématique des caractéristiques de fonctionnement d'une pompe (ou pompe turbine) centrifuge. -... Courbe souhaitée et imposée dans la majorité des contrats de pompe.

naissance de ce problème permettra d'accroître la compétitivité de l'industrie française sur le marché international de ces machines, actuellement en extension.

Aussi, dans le cadre de ses travaux, le groupe de travail concerné s'est donné entre autres tâches celle d'entreprendre l'approche par le calcul du "débit critique" et la comparaison de ce calcul avec les résultats expérimentaux.

\section{Calculs envisageables}

Si de nombreuses méthodes de calcul existent actuellement qui permettent de prédire l'écoulement interne du fluide dans ces turbomachines en se rapprochant sensiblement de la réalité, elles ont toutes trait au régime optimal.

Il n'existe pas, à notre connaissance, de théorie capable de décrire l'écoulement du fluide dans les zones fortement désadaptées.

En effet, les études théoriques et expérimentales très poussées effectuées sur les turbomachines à fluides compressibles (compresseurs, turbines à vapeur ou à gaz) ont permis de montrer qu'il était toujours très difficile d'obtenir une bonne corrélation théorieexpérience pour des régimes se situant loin du fonctionnement optimal.

Dans une $1^{\dot{e} r e}$ étape le calcul serait fait en fluide parfait (voir Fig. 2).

A l'heure actuelle plusieurs méthodes de calcul sont connues et un certain nombre de programmes existent, et sont opérationnels. On pourrait tenter de tirer une information de l'extension de ces calculs au-delà de leur limite actuelle d'utilisation. 


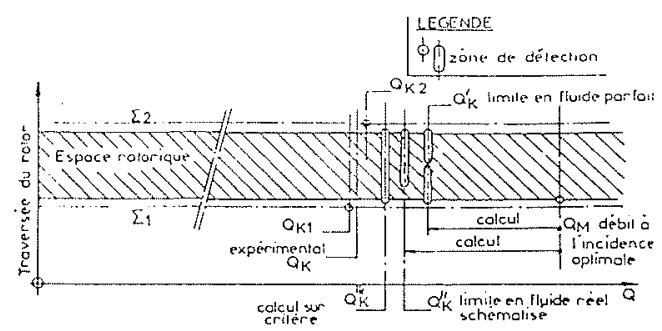

Figure 2 - Illustration schématique des différentes approches du débit critique.

Dans une $2^{e}$ étape ultérieure on s'efforcerait d'introduire dans le calcul d'une façon plus ou moins empirique, la présence des couches limites et des pertes, d'une manière analogue à ce qui a pu être fait pour les compresseurs axiaux, et ceci si possible en liaison avec un organisme spécialisé dans ces questions (voir Fig. 2).

Dans une $3^{e}$ étape (ou peut être en même temps que les précédentes) on ferait l'inventaire des différents critères utilisés, combinant des paramètres géométriques et hydrauliques, critères dont la valeur calculée peut permettre de déterminer dans un certain nombre de cas une valeur approchée du débit critique. On s'efforcerait d'améliorer ces critères et de préciser leurs limites d'application (voir Fig. 2).

\section{Essais expérimentaux envisageables}

Il s'agit entre autres d'une détection de l'apparition de recirculations, soit à l'entrée (traversée de la surface $\Sigma_{1}$, figure 3 ) soit à la sortie (traversée de la surface $\Sigma_{2}$, figure 3 ) d'une roue centrifuge.

Les environnements amont et aval de la roue seront conçus et étudiés pour respecter au mieux les hypothèses de calcul sauf évidemment les couches limites,

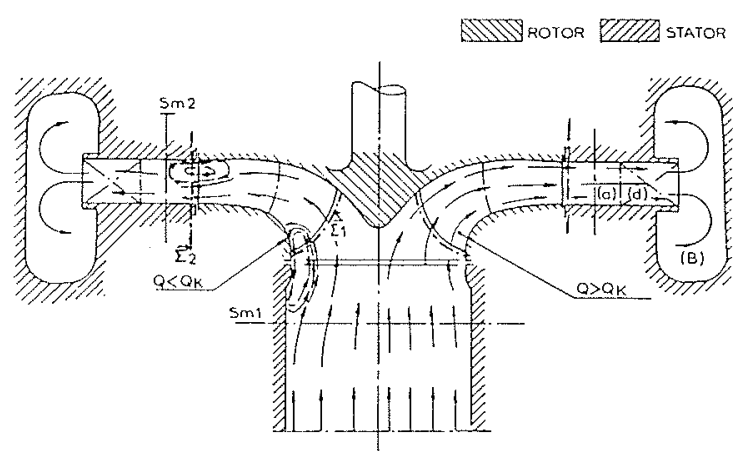

Figure $3-$ Coupe schématique d'un écoulement dans une pompe centrifuge.

(a) : Espace annulaire aval de mesure

(d) : Dispositif statorique aval

B : Bâche collectrice

$\Sigma_{1}$ : Section de détection amont

$\Sigma_{2}$ : Section de détection aval

$\mathrm{Sm}_{1}$ : Section de mesure et d'exploration amont

$\mathrm{Sm}_{2}$ : Section de mesure et d'exploration aval.

pertes et écoulements secondaires inévitables. On cherchera en particulier à éliminer l'influence de l'organe statorique aval de la roue et des fuites.

Toute information et toutes mesures sur les écoulements à l'intérieur de la roue et dans les sections de mesure amont et aval $\mathrm{Sm}_{1}$ et $\mathrm{Sm}_{2}$ (voir Fig. 3) ainsi que sur la valeur des pertes en divers points à l'approche du débit critique sera très utile.

Les essais pourront être faits :

- soit à l'air, en circuit ouvert avec toutes les facilités que cela comporte (emploi de fils chauds ou de films chauds, etc.) mais en évitant les risques de pompage ; - soit à l'eau en circuit fermé pressurisé pour que la cavitation ne soit pas une gêne mais au contraire un moyen de détection grâce à la "pointe" caractérisée de la courbe de cavitation au "débit critique". 